\title{
Seqüência de transmissões não habituais da infecção chagásica em uma mesma família: transfusional para a mãe e congênita para o filho, de cepa de Trypanosoma cruzi resistente ao tratamento
}

\author{
Sequence of unusual Chagas infection transmissions in the same family: \\ mother by blood transfusion and child congenitally, with a \\ treatment-resistant strain of Trypanosoma cruzi
}

\begin{abstract}
Abilio Augusto Fragata Filho', Edileide de Barros Correia', Renato Borges Filho', Marcos de Oliveira Vasconcelos ${ }^{1}$, Daniel Janczuk ${ }^{1}$ e Cícero Soares de Souza Martins ${ }^{1}$
\end{abstract}

\begin{abstract}
RESUMO
Os autores apresentam dois casos de transmissão não habitual da infecção chagásica em uma mesma família: a mãe, por transfusão sangüínea e seu segundo filho, via transplacentária. 0 diagnóstico da criança foi acidental e a resposta ao tratamento etiológico, em ambos, foi ineficaz em erradicar a parasitemia. Atualmente, mãe e filho permanecem em forma indeterminada da doença.
\end{abstract}

Palavras-chaves: Doença de Chagas. Doença de Chagas congênita. Doença de Chagas transfusional.

\section{ABSTRACT}

The authors present two cases of unusual transmission of Chagas infection in the same family: the mother by blood transfusion and her second child across the placenta. The child was diagnosed by chance and etiological treatment for both the mother and the child was ineffective in eradicating the parasitemia. At present, they continue to present the indeterminate form of the disease.

Key-words: Chagas disease. Congenital Chagas disease. Transfusional Chagas disease.

A doença de Chagas ainda é uma das mais importantes endemias da América Latina. Apesar de sua importância, ela é pouco lembrada quando se analisa pacientes fora das áreas endêmicas, ou que não têm epidemiologia para a transmissão vetorial. Muito embora o Brasil tenha recebido em 2006 o certificado de eliminação da transmissão através do Triatoma infestans, devemos lembrar dos outros mecanismos, dos quais o congênito é pouco considerado, face à sua baixa incidência. Poucos são os Serviços de Pré-Natal que incluem, rotineiramente, exame do soro, para doença de Chagas, em gestantes procedentes ou naturais de áreas endêmicas para esta enfermidade.

\section{RELATO DE CASO}

Criança do sexo masculino, nascida em São Paulo, SP, em 16 de fevereiro de 1994, de parto cesáreo por descolamento prematuro de placenta, com $3.000 \mathrm{~g}$ e $48 \mathrm{~cm}$. No berçário foi identificada hepato-esplenomegalia e no hemograma houve o encontro de um Trypanosoma cruzi.

Tratava-se de segundo filho de casal jovem (o primeiro filho normal), sendo que a mãe havia contraído doença de Chagas pós-transfusão sanguínea (pós-operatório de dermolipectomia)

\footnotetext{
1. Setor de Miocardiopatias, Instituto Dante Pazzanese de Cardiologia, São Paulo, SP.

Endereço para correspondência: Dr. Abilio Augusto Fragata Filho. Setor de Miocardiopatias/Instituto Dante Pazzanese de Cardiologia. Av. Dante Pazzanese 500, Ibirapuera, 04012-190 São Paulo, SP.

Tel: 5511 5085-6055; Fax: 5511 5087-9596

e-mail: a.frag@terra.com.br

Recebido para publicação em: 13/08/2007

Aceito em: 06/11/2007
} 
aos 18 anos de idade (década de 70), sendo o diagnóstico feito por apresentar febre por volta da segunda semana pós-transfusão e ter exame de sangue periférico com encontro de Trypanosoma cruzi. Nesta época fora tratada com benzonidazol $10 \mathrm{mg} / \mathrm{kg} / \mathrm{dia}$ por 60 dias, porém, permaneceu com hemoculturas e sorologias positivas, em controle clínico e em forma indeterminada da doença.

Iniciou-se o tratamento do recém-nascido aos 11 dias de vida, com benzonidazol 7,5mg/kg/dia por 60 dias. Após completado este esquema terapêutico, foi realizada hemocultura que resultou positiva. Nova série de benzonidazol, agora com $10 \mathrm{mg} / \mathrm{kg} /$ dia por 60 dias foi prescrita, no entanto, a hemocultura posterior permaneceu positiva. Foi prescrito nifurtimox na dose de $15 \mathrm{mg} / \mathrm{kg} / \mathrm{dia}$, por 60 dias, mas os efeitos colaterais como anorexia e convulsão obrigaram à suspensão deste fármaco. Optou-se então por mantê-lo em observação clínica, pela inexistência de outras opções. Em última consulta, que foi realizada em junho de 2007, a criança com 13 anos mantinha-se assintomática, sem alterações do exame físico, com eletrocardiograma, radiografia de tórax e ecocardiograma normais, sem queixas digestivas. Sua mãe igualmente se mantinha em forma indeterminada. Ambos mantêm exame do soro positivo para doença de Chagas (ELISA e imunofluorescência).

\section{DISCUSSÃO}

A transmissão da infecção chagásica tem ocorrido, ao longo da história, preferencialmente pela forma vetorial. Campanhas sérias de erradicação do Triatoma infestans, principal agente transmissor, conseguiram minimizar a incidência da enfermidade em todo 0 território nacional ${ }^{413}$. A transfusão de sangue contaminado pelo Trypanosoma cruzi foi a segunda forma de transmissão desta doença em prevalência, porém, o término da doação remunerada e o controle do sangue a ser transfundido que obrigou os serviços de hemoterapia a realizarem ao menos duas técnicas sorológicas no sangue colhido (Portaria 1376, Ministério da Saúde, 19/11/93) tornaram esta possibilidade pouco provável nos dias de hoje. Na forma transfusional de transmissão, a febre é frequiente, sendo importante lembrar desta possibilidade em paciente transfundido que apresenta febre, com períodos pós-transfusão que variam de cerca de 1 semana a 4 meses $^{5814}$. 0 diagnóstico se dá por levantamento epidemiológico, pesquisa direta de parasita no sangue periférico, ou por sorologia (IgM, e/ou $\operatorname{IgG}$ ). Estabelecido o diagnóstico, o tratamento parasiticida é imperioso ${ }^{6}{ }^{12}$. A mãe desta criança apresentou o quadro clínico muito sugestivo da transmissão transfusional, febre na segunda semana e parasitas no sangue periférico e a criança, nasceu com hepato-esplenomegalia e houve encontro ocasional de parasita em sangue periférico.

A transmissão congênita foi suspeitada por Carlos Chagas em 1911, mas somente foi confirmada em humanos no ano de 1949 por Dao, na Venezuela ${ }^{10}$.

É possível que gestantes chagásicas em fase aguda tenham maior probabilidade de transmitir a infecção, haja vista a intensa parasitemia nestes casos. Poucas são as descrições de grávidas em fase aguda, não havendo obrigatoriamente relação entre eles e maior probabilidade de transmissã $0^{9}$.
$\mathrm{Na}$ fase crônica, a baixa parasitemia poderia minimizar as chances de transmissão transplacentária, haja vista a desproporção entre a prevalência de gestantes com sorologia positiva para Chagas e os recém-nascidos contaminados ${ }^{13}$. A atenção à gestante chagásica deveria ser normatizada pelas autoridades de Saúde Pública, exigindo-se a investigação sorológica de toda a grávida com epidemiologia positiva para esta enfermidade, estendendo-se esta investigação aos conceptos, no caso de mães positivas ${ }^{11}$.

Em estudo realizado de 1972 a 1991, Moya e col$^{10}$ analisaram 56.849 gestantes, das quais $8.830(15,5 \%)$ com sorologia positiva para Chagas e nelas encontraram $1,4 \%$ de mal formações congênitas, ao passo que nas 48.019 (84,5\%) das não chagásicas houve 3\% destas mal formações, evidenciando não haver relação entre mães com doença de Chagas e má formação fetal'5. As crianças acometidas de forma congênita podem ser classificadas em assintomáticas e sintomáticas. Quando há sintomas, estes podem ser precoces ou tardios, quer ocorram no primeiro mês de vida ou mais tardiamente. A hepatomegalia é o sinal mais importante do acometimento neonatal da doença, seguido pela esplenomegalia. Nos casos não tratados, estas visceromegalias regridem em cerca de 6 a 12 meses. Em recém-nascido de mãe chagásica, com hepato-esplenomegalia devemos pensar na transmissão congênita da doença ${ }^{2}$. Outras manifestações podem ser: taquicardia, insuficiência cardíaca, alterações eletrocardiográficas (alterações inespecíficas da repolarização ventricular, aumento do intervalo PR, baixa voltagem dos acidentes eletrocardiográficos), encefalite e meningite . $^{6}$

O diagnóstico da transmissão congênita da doença de Chagas baseia-se no encontro do parasita no sangue circulante do recém nascido, que pode ser obtido por exame a fresco, gota espessa, entre outros, ou exames indiretos, como hemocultura e xenodiagnóstico. O exame sorológico deve ser feito com a pesquisa de IgM (esta pesquisa, porém, oferece dificuldades técnicas, podendo resultar em falso negativo, razão pela qual não é habitualmente realizada) e IgG. Esta última pode ser resultante da passagem da IgG da mãe para o concepto, não significando então a contaminação e que desaparece em cerca de 6 a 9 meses, ou ser a IgG da criança, que não irá desaparecer e sim confirmar a transmissão transplacentária. Assim sendo, quando só se identifica IgG positiva, deve-se repetir o exame em cerca de 6 a 9 meses, para confirmar ou não a doença ${ }^{611}$. Na confirmação da passagem do Trypanosoma cruzi da mãe para o concepto o tratamento parasiticida é mandatório. Em nosso meio ele é realizado com o benzonidazol na dose de 7,5 a 10mg/kg/dia durante 30 a 60 dias, em 2 ou 3 tomadas diárias. Quando disponível, o nifurtimox pode se constituir em outra opção terapêutica, sendo prescrito na dose de 10 a $12 \mathrm{mg} / \mathrm{kg} / \mathrm{dia}$, em 2 ou 3 tomadas diárias, igualmente por 30 a 60 dias $^{12}$.

Em levantamento realizado em nossa Instituição, com o exame de 242 crianças menores que 10 anos, nascidas e residentes em São Paulo (SP), filhas de 145 mães portadoras de doença de Chagas, encontramos somente uma criança (caso aqui relatado) com transmissão transplacentária indiscutível $(0,4 \%)$.

No caso apresentado, chama a atenção a seqüência de transmissões não habituais da infecção em uma mesma família, inicialmente a transfusional da mãe, seguida pela transplacentária. 
O diagnóstico ocasional feito através do encontro do parasita em hemograma para a investigação de hepato-esplenomegalia nos mostra que, mesmo em centros avançados do país, pouco se pensa na doença de Chagas, mesmo sendo ela muito prevalente entre nós.

É importante ressaltar que gestantes com epidemiologia positiva para doença de Chagas devam ter o diagnóstico confirmado por exame do soro e nos casos positivos, o recém nascido merecerá toda a atenção quanto à possível existência da doença congenitamente transmitida e nos casos positivos, 0 tratamento parasiticida deve ser imediatamente providenciado.

É lamentável que, no Brasil, só dispomos de um fármaco (benzonidazol), com ação parasiticida demonstrada, o que restringe muito nosso campo de ação no tratamento destes casos, onde consensualmente se indica o tratamento etiológico da doença de Chagas. Nestes dois pacientes, mãe e filho, o benzonidazol não teve a ação parasiticida desejada, seguramente por resistência deste parasita à ação deste medicamento e o nifurtimox não foi tolerado pela criança, nos deixando sem outras opções terapêuticas.

0 Consenso Brasileiro em Doença de Chagas ${ }^{7}$, da Sociedade Brasileira de Medicina Tropical, apresenta um fluxograma (Figura 1) para a condução dos recém nascidos de gestantes com doença de Chagas.

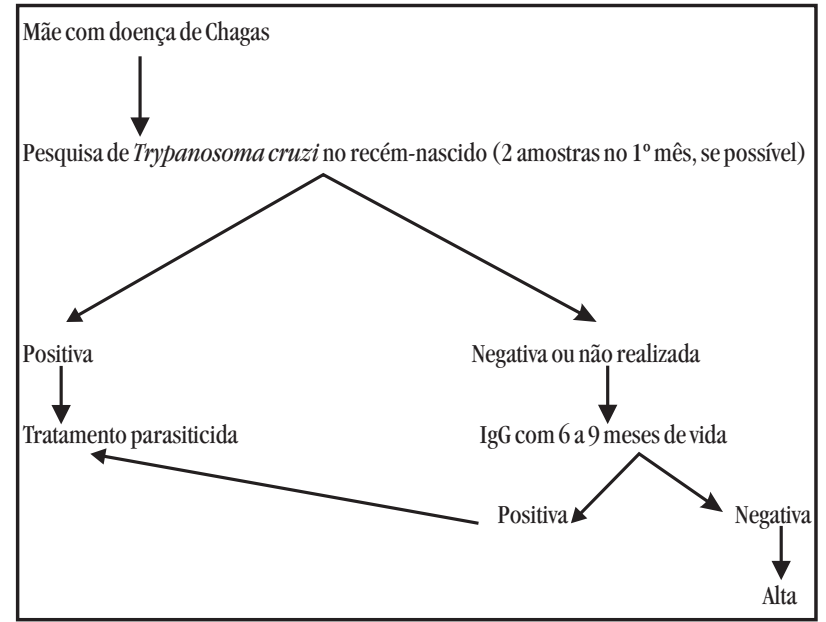

Figura 1 - Fluoxograma de acompanbamento de recém-nascido de mãe com doença de Chagas (Adaptado: Consenso Brasileiro em Doença de Chagas. Rev Soc Bras Med Tropical vol 38 (supl III): 1-29, 2005).

\section{REFERÊNCIAS}

1. Bittencourt AL. Possible risk factors for vertical transmission of Chagas' disease. Revista do Instituto de Medicina Tropical de São Paulo 34: 403-408, 1992.

2. Bittencourt AL. Transmissão vertical da doença de Chagas. Revista de Patologia Tropical 29 (supl): 101-113, 2000.

3. Bittencourt AL, Sadigursky M, Barbosa HS. Doença de Chagas Congênita. Estudo de 29 casos. Revista do Instituto de Medicina Tropical de São Paulo 17:146-159, 1975.

4. Dias JCP, Coura JR. Epidemiologia. In: Dias JCP, Coura JR (orgs) Clinica e Terapêutica da Doença de Chagas: uma abordagem prática para o clínico geral. Editora Fundação Oswaldo Cruz, Rio de Janeiro, p. 33-66, 1997.

5. Dias JCP, Schofield CJ. Controle da transmissão transfusional da doença de Chagas na Iniciativa do Cone Sul. Revista da Sociedade Brasileira Medicina Tropical 31: 373-383, 1998.

6. Luquetti A0, Rassi A. Diagnóstico laboratorial da infecção pelo Trypanosoma cruzi. In: Brener Z, Andrade ZA, Barral Netto M (orgs) Trypanosoma cruzi e doença de Chagas, $2^{a}$ edição, Guanabara Koogan, Rio de Janeiro, p. 344-378, 1999.

7. Ministério da Saúde. Secretaria de Vigilância em Saúde. Consenso Brasileiro em Doença de Chagas, Uberaba, MG. Revista da Sociedade Brasileira Medicina Tropical 38 (supl III): 11-14, 2005.

8. Moraes-Souza H. Transmissão transfusional da doença de Chagas. Revista de Patologia Tropical 29 (supl): 91-100, 2000.

9. Moretti E, Basso B, Castro I, Paez MC, Chaul M, Barbieri G, Feijoo DC, Sartori MJ, Paez RC. Chagas' disease: study of congenital transmission in cases of acute maternal infection. Revista da Sociedade Brasileira Medicina Tropical 38: 53-35, 2005.

10. Moya PR, Moretti EA. Doença de Chagas Congênita. In: Dias JCP, Coura JR (orgs) Clinica e Terapêutica da Doença de Chagas: uma abordagem prática para o clínico geral. Editora Fundação Oswaldo Cruz, Rio de Janeiro, p. 383409, 1997.

11. Nisida IVV. Estudo descritivo da avaliação da transmissão congênita da doença de Chagas em três Instituições da cidade de São Paulo. Revista da Sociedade Brasileira Medicina Tropical 32: 79-80, 1999.

12. Organización Panamericana de la Salud. Tratamiento etiológico de la enfermedad de Chagas: conclusiones de una consulta técnica. Organización Panamericana de la Salud, Washington DC, 1998.

13. Silveira AC, Rezende DF. Epidemiologia e controle da transmissão vetorial da doença de Chagas no Brasil. Revista da Sociedade Brasileira Medicina Tropical 27 (supl III): 11-22, 1994.

14. Wendell S. Doença de Chagas transfusional. In: Dias JCP, Coura JR (orgs) Clinica e Terapêutica da Doença de Chagas: uma abordagem prática para o clínico geral. Editora Fundação Oswaldo Cruz, Rio de Janeiro, p. 411-427, 1997. 